\title{
Quadrupole Deformed and Octupole Collective Bands in ${ }^{228} \mathrm{Ra}$
}

\author{
K. Gulda ${ }^{1)}$, H. Mach $^{2)}$, A.J. Aas ${ }^{3)}$, M.J.G. Borge ${ }^{4)}$, D.G. Burke ${ }^{5)}$, B. Fogelberg ${ }^{2)}$,
} H. Gietz ${ }^{6)}$, I.S. Grant ${ }^{7)}$, E. Hagebo ${ }^{3)}$, P. Hill ${ }^{8)}$, P. Hoff ${ }^{3)}$, N. Kaffrell ${ }^{6, *)}$, W. Kurcewicz ${ }^{1}$, A. Lindroth ${ }^{2)}$, G. Løvhøiden ${ }^{8)}$, T. Martinez $^{9)}$, S. Mattsson ${ }^{10)}$, R.A. Naumann ${ }^{11)}$, K. Nyb $\phi^{8)}$, G. Nyman ${ }^{10)}$, B. Rubio ${ }^{9)}$, M. Sanchez-Vega ${ }^{2)}$, J.L. Tain ${ }^{9)}$, R. B. E. Taylor ${ }^{2)}$, O. Tengblad ${ }^{12,4)}$, T.F. Thorsteinsen ${ }^{13)}$, and the ISOLDE Collaboration.

\begin{abstract}
Spins and parities for collective states in ${ }^{228} \mathrm{Ra}$ have been determined from conversion electron measurements with a mini-orange $\beta$ spectrometer. The fast-timing $\beta \gamma \gamma(t)$ method has been used to measure lifetimes of $\mathrm{T}_{1 / 2}=550(20)$ ps and 181(3) ps for the $2_{1}^{+}$and $4_{1}^{+}$members of the $\mathrm{K}=0^{+}$band, and $\mathrm{T}_{1 / 2} \leq 7 \mathrm{ps}$ and $\leq 6 \mathrm{ps}$ for the $1_{1}^{-}$and $3_{1}^{-}$members of the $\mathrm{K}=0^{-}$band, respectively. The quadrupole moments, $Q_{0}$, deduced from the $\mathrm{B}\left(\mathrm{E} 2 ; 2_{1}^{+} \rightarrow 0_{1}^{+}\right)$and $\mathrm{B}\left(\mathrm{E} 2 ; 4_{1}^{+} \rightarrow 2_{1}^{+}\right)$rates are in a good agreement with the previously measured value and the systematics of the region. However, the $\mathrm{B}(\mathrm{E} 1)$ rates of $\geq 4 \times 10^{-4} \mathrm{e}^{2} \mathrm{fm}^{2}$, which represent the first $\mathrm{B}(\mathrm{E} 1)$ measurements for this nucleus, are at least 25 times larger than the value previously suggested for ${ }^{228} \mathrm{Ra}$. The new results are consistent with the $\mathrm{B}(\mathrm{E} 1)$ rates recently measured for the neighbouring ${ }^{227} \mathrm{Ra}$ and reveal octupole correlations in ${ }^{228} \mathrm{Ra}$.
\end{abstract}

(IS322)

(Accepted for publication in Nuclear Physics A)

\footnotetext{
1) Dept. of Physics, University of Warsaw, Pl-00 681 Warsaw, Poland

Dept. of Neutron Research, University of Uppsala, S-61182 Nyköping, Sweden

3) Dept. of Chemistry, Univ. of Oslo, P.O.Box 1033, Blindern, N-0315 Oslo, Norway

4) Insto. De Estructura de la Materia, CSIC, E-28006 Madrid, Spain

5) Dept. of Physics, McMaster Univ., Hamilton, Ontario, L8S 4K1, Canada

6) Institut fur Kernchemie, Universitet Mainz, D-55099 Mainz, Germany

7) The Schuster Laboratory, Manchester University, Manchester M13 9PL, UK

8) Dept. of Physics, Univ. of Oslo, P.O.Box 1048, Blindern, N-0316 Oslo, Norway

9) Insto. de Física Corpuscular, CSIC-Univ. Valencia, E-46100 Burjassot, Spain

10) Dept.of Physics, Chalmers Univ. of Technology, S-41296 Göteborg, Sweden

11) Dept.of Physics, Dartmouth College, Hanover, NH 03755, USA

12) PPE Division, CERN, CH-1211 Geneva 23, Switzerland

13) Department of Physics, University of Bergen, N-5007 Bergen, Norway

*) Deceased
} 
Introduction

${ }^{228} \mathrm{Ra}$ is situated in the upper mass border region of the "island of octupole deformation" where the octupole deformation is vanishing in the presence of a large quadrupole field and the octupole collectivity is revealed by octupole vibrations. Two of our recent studies have been focussed on the odd-A nuclei in this region: ${ }^{227} \mathrm{Fr}$ [1] and ${ }^{227} \mathrm{Ra}$ [2] which have shown properties consistent with a small $\beta_{3}$ and an octupole vibrational interpretation. Several authors (see, for example, the review given in ref. [3]) have investigated the octupole degree of freedom in the even-A Ra nuclei. Egido and Robledo [4, 5] have identified ${ }^{228} \mathrm{Ra}$ as an octupole vibrational nucleus. In contrast, Möller et al. [6] have predicted ground state octupole deformation of $\left|\beta_{3}\right|=0.058$ for ${ }^{228} \mathrm{Ra}$. The aim of this work is twofold: to determine the spin and parity assignments to the low-lying bands via conversion electron measurements, and to clarify the quadrupole and octupole collectivities in this nucleus via a precise determination of the $\mathrm{E} 2$ and $\mathrm{E} 1$ transition rates.

Previous investigation of the low-lying levels of ${ }^{228} \mathrm{Ra}$ performed by Ruchowska et al. [7] and van den Berg et al. [8], produced a detailed level scheme of ${ }^{228}$ Ra. However, the spin-parity assignments given in [7] were rather tentative and based on a comparison with the lighter isotopes of ${ }^{224,226} \mathrm{Ra}$ and on the analysis of reduced branching ratios. The present work not only clarifies the spin/parity assignments but also explores the issue of highly converted transitions with strong E0 components, which were first suggested by Ruchowska and collaborators [7]. Evidence for a strongly excited $0^{+}$state at $\mathrm{E}=0.8 \mathrm{MeV}$ in ${ }^{228} \mathrm{Ra}$ has been found in the ${ }^{232} \mathrm{Th}\left(\mathrm{d},{ }^{6} \mathrm{Li}\right){ }^{228} \mathrm{Ra}$ reaction [8].

Ahmad et al. [9] have examined the laser spectroscopic results for ${ }^{208-232} \mathrm{Ra}$ in terms of evidence for octupole instability. In particular, they have compared two data sets: the first represented the deformed part deduced from the isotope shifts, while the second represented droplet model calculations that accounted only for the even multipole deformation. The difference between these sets was taken as evidence for the existence of a sizeable octupole contribution near ${ }^{224} \mathrm{Ra}$. In this analysis, ${ }^{228} \mathrm{Ra}$ represented a crucial renormalization point between data sets, since it was the heaviest Ra nucleus for which the $\beta_{2}$ deformation parameter was known (and obtained from the experimental $\mathrm{B}(\mathrm{E} 2$; $2_{1}^{+} \rightarrow 0_{1}^{+}$) rate [10]). Unfortunately, a large statistical uncertainty associated with this renormalization point made it difficult to draw any firm conclusion on the size of the $\beta_{3}$ contibution. The present study improves the accuracy of the $\mathrm{B}(\mathrm{E} 2)$ value by a factor of 2 .

The $\mathrm{B}(\mathrm{E} 1)$ rates for transitions connecting the lowest-lying $\mathrm{K}=0^{ \pm}$bands represent another important characteristic which needs to be clarified for ${ }^{228} \mathrm{Ra}$. The results of theoretical predictions vary quite strongly. The microscopic calculations by Egido and Robledo [4, 5] predict fast $\mathrm{B}(\mathrm{E} 1)$ rates for this nucleus, $\approx 3-7 \times 10^{-3} \mathrm{e}^{2} \mathrm{fm}^{2}$, driven by the octupole vibrational mode of excitation, while the calculations by Denisov [11] yield such a high rate for ${ }^{226} \mathrm{Ra}$, but not for ${ }^{228} \mathrm{Ra}$, where he predicts a dramatic drop of the $\mathrm{E} 1$ rates to $\mathrm{B}(\mathrm{E} 1) \approx 4 \times 10^{-4} \mathrm{e}^{2} \mathrm{fm}^{2}$.

At the other extreme are the schematic model calculations of Butler and Nazarewicz [12] which predict dipole moments in the reflection-asymmetric nuclei within the shell correction method (which, unfortunately, as noted by the authors, does not properly include the dynamical shape-fluctuations). These authors provide no estimate for ${ }^{228} \mathrm{Ra}$, but within the framework of the model they have used (the values of $\beta$ parameters are from calculations [13]), the dipole moment would have to be zero, as ${ }^{228} \mathrm{Ra}$ is the first heavy Ra nucleus predicted [13] to be reflection symmetric. The relevance of the schematic model to the case of ${ }^{228} \mathrm{Ra}$ rests with a respectable agreement of the model predictions over 
extensive systematics of the experimental E1 moments, and in particular this agreement includes ${ }^{227} \mathrm{Ra}$ - the very neighbour of ${ }^{228} \mathrm{Ra}$. In fact, the latter point of agreement was established only recently in our measurements [2]. Following the trend of the E1 moment systematics and the schematic model predictions one would expect to find a particularly low $\mathrm{B}(\mathrm{E} 1)$ rate in ${ }^{228} \mathrm{Ra}$. Indeed, ref. [12] lists $D_{0}=0.011(1)$ e.fm as an experimental value for ${ }^{228} \mathrm{Ra}$. It gives a $\mathrm{B}(\mathrm{E} 1)$ rate of about $1.4 \times 10^{-5} \mathrm{e}^{2} \mathrm{fm}^{2}$, which is 500 times lower than the value predicted by Egido and Robledo [4, 5]. If correct, such result would challenge the latter calculations.

However, the low $\mathrm{E} 1$ rate in ${ }^{228} \mathrm{Ra}$ did not represent a measured value but merely a guess. It was based on a hypothesis [7] that in the ${ }^{228} \mathrm{Fr} \rightarrow{ }^{228} \mathrm{Ra}$ decay there is no $\beta^{-}$ feeding to the $1_{1}^{-}$state. Then the $\mathrm{B}(\mathrm{E} 1)$ rate was derived from the intensity ratio of the E1 and E2 transitions de-exciting the $3_{1}^{-}$level, where the unobserved E2 branch was estimated from a difference between the observed transition intensity de-exciting the $1^{-}$ state and the one feeding it. This hypothesis has not been supported, however, by a nuclear magnetic resonance measurement [14] where a spin/parity of $2^{-}$was established for the ground state of ${ }^{228} \mathrm{Fr}$. Thus contrary to the hypothesis, a direct $\beta^{-}$decay to the $1^{-}$state is, in fact, quite possible. The procedure described above can only yield a lower limit, but not the absolute value, of $D_{0}$ in ${ }^{228} \mathrm{Ra}$. Consequently, the value quoted in ref. [12] should be corrected to become a lower limit of $D_{0} \geq 0.01 \mathrm{e} \cdot \mathrm{fm}$ which is not very informative. A $\mathrm{B}(\mathrm{E} 1)$ rate for ${ }^{228} \mathrm{Ra}$ is yet to be measured.

A recent introduction of the fast timing $\beta \gamma \gamma(\mathrm{t})$ method to the PSB ISOLDE mass separator at CERN [15] has opened the heavy actinides to the direct lifetime measurements in the low picosecond range. We have applied this method to provide precise level lifetimes for the critical $\mathrm{K}=0^{+}$and $\mathrm{K}=0^{-}$bands in ${ }^{228} \mathrm{Ra}$ as part of our systematic studies of the level lifetimes in the ${ }^{222-230} \mathrm{Ra}$ isotopes. Preliminary results from the conversion electron measurements have been reported in conference proceedings [16]. These results are superseded by the present findings.

\section{$2 \quad$ Experimental methods and results}

\subsection{Source preparation}

Two sets of conversion electron measurements have been performed. The first measurement, characterized by higher statistics, was done at the ISOLDE II facility. Sources of ${ }^{228} \mathrm{Fr}$ were produced in a spallation reaction of ${ }^{238} \mathrm{UC}_{2}$ induced by $600 \mathrm{MeV}$ protons from the CERN Synchrocyclotron and mass-separated in the on-line isotope separator. The activity was deposited on a tape transport system and carried to a well-shielded counting station.

For the $\beta \gamma \gamma(\mathrm{t})$ and the second conversion electron measurements, the francium activity was produced by a spallation reaction in a ${ }^{232} \mathrm{ThC}_{2}$ target, induced by $1 \mathrm{GeV}$ protons from the CERN PS-Booster. The ions were mass-separated in the ISOLDE online separator and deposited onto a tape transport system. The fast timing measurements were performed at the point of beam deposition, while the conversion electrons were measured at a separate counting station positioned about $100 \mathrm{~cm}$ away.

\subsection{Electron conversion coefficients}

Multipolarities of prominent transitions in ${ }^{228} \mathrm{Ra}$ have been established by a simultaneous measurement of conversion electrons in the mini-orange spectrometer [17] and $\gamma$-rays in a Ge detector. The electron spectrometer combined a liquid-nitrogen cooled 
$\mathrm{Si}(\mathrm{Li})$ detector (characterized by $300 \mathrm{~mm}^{2}$ area, $2 \mathrm{~mm}$ depletion depth and $2.0 \mathrm{keV}$ resolution) with a filter of permanent magnets around a central absorber. Precisely known transition energies from ${ }^{228} \mathrm{Fr}[7]$ were used as internal energy standards. The transmission curve of the mini-orange spectrometer was obtained using conversion lines from sources of ${ }^{133} \mathrm{Ba},{ }^{152} \mathrm{Eu}$ and ${ }^{207} \mathrm{Bi}$. The two sets of the conversion measurements were performed using similar setup and geometry but different Si(Li) detectors. The second measurement, which was characterized by significantly lower statistics, had much improved energy resolution. Figure 1 shows the conversion electron spectrum from the decay of ${ }^{228} \mathrm{Fr}$ to ${ }^{228} \mathrm{Ra}$ obtained in the first measurement. A key section of the electron spectrum obtained in the second run is shown as an insert to this figure. It covers a small energy region near the $\mathrm{K}$ conversion peak of the $259 \mathrm{keV}$ E0 transition.

Multi-spectra measurements were performed in the first run to verify whether the stronger conversion lines belong to the ${ }^{228} \mathrm{Fr}$ decay. The observed half-lives for the $64 \mathrm{keV}$ $\mathrm{L}$ and $\mathrm{M}$ lines, $140 \mathrm{keV} \mathrm{M}$ line, the composite-peak (which included the $171 \mathrm{keV} \mathrm{L}$ and $259 \mathrm{keV} \mathrm{K}$ lines), $676 \mathrm{keV} \mathrm{K}$ line, $707 \mathrm{keV} \mathrm{K}$ line, and the $721 \mathrm{keV} \mathrm{K}$ line were found in agreement with the expected value [7] of 38(1) s.

In the first step, we have established the $\mathrm{E} 2$ multipolarity for the $63.8 \mathrm{keV} 2^{+} \rightarrow 0^{+}$ and $140.9 \mathrm{keV} 4^{+} \rightarrow 2^{+}$transitions from the analysis of the experimental conversion electron subshell ratios. For the $63.8 \mathrm{keV}$ transition the electron intensity ratios of the $\left(\mathrm{L}_{1}+\mathrm{L}_{2}\right)$ $/ \mathrm{L}_{3} / \mathrm{M} /(\mathrm{N}+\ldots)$ lines have been measured as: $100 / 83.5(23) / 60(4) / 18.7(20)$ which closely follow the theoretical [18] values of $100 / 82.5 / 50$ / 15.6 for E2 multipolarity. For the $140.9 \mathrm{keV}$ transition the experimental and theoretical E2 intensity ratios are $100 /$ $57.4(34) / 39.6(27) / 13.6(16)$ and $100 / 56.9 / 37.7 / 15.1$, respectively, thus also indicating E2 multipolarity. In the second step, we have adopted the theoretical E2 conversion coefficient of $\alpha_{M+N+\ldots}=0.507$ for the $140.9 \mathrm{keV}$ transition which allowed us to renormalize the relative electron and gamma [7] intensities and evaluate the conversion coefficients for other observed transitions. A summary of the conversion electron results is presented in table 1. In particular, we have established two new E0 transitions and identified three mixed $\mathrm{E} 0+\mathrm{M} 1+\mathrm{E} 2$ transitions with a major $\mathrm{E} 0$ component, which have been placed in the decay scheme of ${ }^{228} \mathrm{Fr}$.

In our early analysis [16] the electron peak at $\sim 155 \mathrm{keV}$ has been interpreted as a single peak due to the $171.4 \mathrm{keV} \mathrm{L}$ transition. However, the electron spectrum from the second measurement shows this peak is a doublet with two distinctive components (see an insert in Fig. 1): the $\mathrm{L}$ conversion peak of the $171.4 \mathrm{keV}$ transition and the $\mathrm{K}$ conversion peak of a new $259.9 \mathrm{keV}$ E0 line, respectively. For the $171.4 \mathrm{keV}$ transition, we have obtained an experimental $\mathrm{L}_{1}+\mathrm{L}_{2}$ conversion coefficient equal to $1.0(3)$, which when compared to the theoretical values of $0.017,0.41$ and 0.53 for the E1, E2 and M1 multipolarities, respectively, revealed a significant E0 component. The contribution from the $\mathrm{L}_{1}+\mathrm{L}_{2}$ line of the $172.4 \mathrm{keV}$ transition has been subtracted by taking the theoretical $\mathrm{E} 2$ conversion coefficient for this transition (the electron intensity of the $\mathrm{L}_{3}$ line excludes a higher multipolarity for the $172.4 \mathrm{keV}$ line).

\subsubsection{9-keV E0 transition}

The $156.0 \mathrm{keV}$ electron peak, which overlaps with the $\mathrm{L}_{3}$ line of the $171.4 \mathrm{keV}$ transition, is interpreted as the $\mathrm{K}$ line of the $259.9 \mathrm{keV}$ transition. An experimental lower limit of 17 has been obtained for its $\mathrm{K}$ conversion coefficient indicating an E0 character. An upper limit of 0.3 was established for the relative $\gamma$ intensity of the $259.9 \mathrm{keV}$ transition 

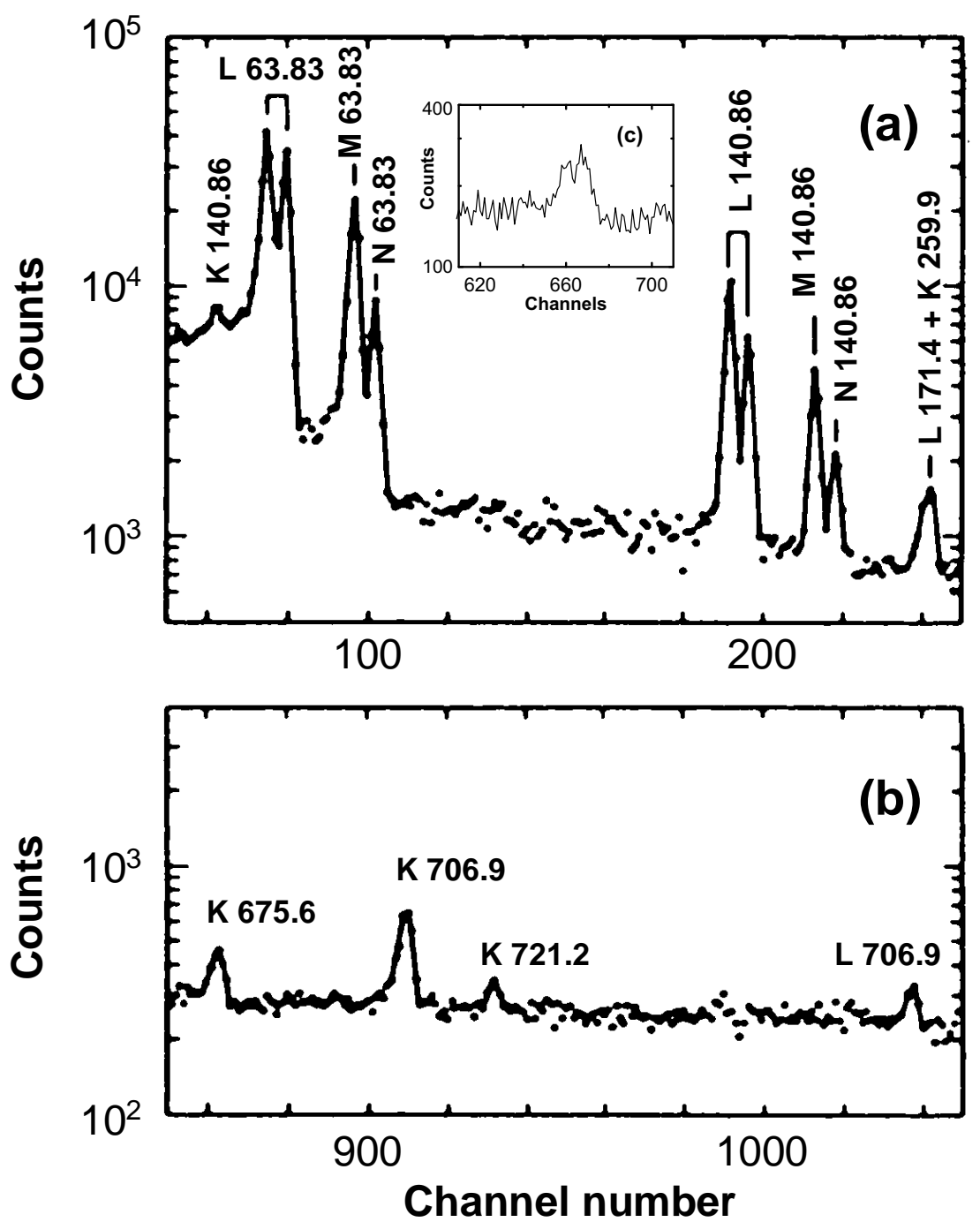

Figure 1: Electron spectrum from the decay of ${ }^{228} \mathrm{Fr}$ to ${ }^{228} \mathrm{Ra}$ : (a) the lower-energy part, (b) the higher-energy part. Peaks are labelled by the transition energy. The insert (c) shows the composite peak, which includes the $171.4 \mathrm{keV} \mathrm{L}$ and $259.9 \mathrm{keV} \mathrm{K}$ peaks, measured with much improved resolution in the second run. 
Table 1: The relative intensity of $\mathrm{K}$ conversion lines, $\mathrm{I}_{K}$, internal conversion coefficients, $\alpha_{K}$, and multipolarity assignments for transitions in ${ }^{228} \mathrm{Ra}$.

\begin{tabular}{cccccccc}
\hline $\begin{array}{c}\text { Transition } \\
\text { energy } \\
(\mathrm{keV})\end{array}$ & $\begin{array}{c}\text { Initial } \\
\text { level } \\
(\mathrm{keV})\end{array}$ & $\mathrm{I}_{K}$ & $\begin{array}{c}\text { Experimental } \\
\alpha_{K}\end{array}$ & & $\begin{array}{c}\text { Theoretical }^{b)} \\
\alpha_{K} \\
\text { M1 }\end{array}$ & E2 & $\begin{array}{c}\text { Multi- } \\
\text { polarity }\end{array}$ \\
\hline $63.83(2)$ & 63.8 & & & & & & E2 ${ }^{c)}$ \\
$140.86(2)$ & 204.7 & $540(80)$ & $0.27(5)$ & 0.17 & 5.0 & 0.29 & E2 \\
167.1 & 1013.3 & & & & & & E0 $\left.{ }^{d}\right)$ \\
$171.4(1)$ & 1070.3 & & & & & & E0+M1+E2 ${ }^{e)}$ \\
$259.9(5)$ & & $103(20)$ & $\geq 17$ & 0.039 & 0.89 & 0.096 & E0 \\
$332.91(5)$ & 537.6 & $31(5)$ & $0.032(7)$ & 0.023 & 0.45 & 0.058 & E1 \\
$410.40(6)$ & 474.1 & $41(5)$ & $0.017(3)$ & 0.015 & 0.26 & 0.038 & E1 \\
$675.6(5)$ & 880.3 & $56(5)$ & $1.3(3)$ & 0.0054 & 0.068 & 0.014 & E0+M1+E2 \\
$706.9(1)$ & 770.7 & $100(6)$ & $0.55(8)$ & 0.0049 & 0.061 & 0.013 & E0+M1+E2 \\
$721.2(5) f)$ & 721.2 & $16(5)$ & $\geq 1.4$ & 0.0048 & 0.057 & 0.013 & E0 \\
$782.3(1)$ & 846.2 & $9(4)$ & $0.012(5)$ & 0.0041 & 0.046 & 0.010 & E2+M1 \\
\hline \hline
\end{tabular}

a) Transition energies from ref. [7] unless stated otherwise.

b) From ref. [18].

c) From the electron intensity ratios of the $\left(L_{1}+L_{2}\right) / L_{3} / \mathrm{M} /(\mathrm{N}+\ldots)$ lines (see subsec. 2.2).

d) From ref. [7].

e) From the electron intensity of the $\left(\mathrm{L}_{1}+\mathrm{L}_{2}\right)$ line (see subsec. 2.2).

f) Transition energy obtained from the conversion electron spectrum.

(relative to $\mathrm{I}_{\gamma} \equiv 100$ for $\mathrm{E}_{\gamma}=140.86 \mathrm{keV}$ ). Very high conversion coefficient and lack of any corresponding $\gamma$ transition implies a pure E0 character for the $259.9 \mathrm{keV}$ transition. This is further supported by its experimental intensity ratio of the $\mathrm{K} /\left(\mathrm{L}_{1}+\mathrm{L}_{2}\right)$ conversion coefficients of 4.3(14), which should be compared to the theoretical value of 5.30 for a pure E0 multipolarity. Care was taken to check whether any in-beam or isobaric impurity could affect this analysis and none was found.

The $259.9 \mathrm{keV}$ transition is an intense E0 line with a total intensity of $\sim 6$ on the scale of $\mathrm{I}_{\gamma}^{140.86} \equiv 100$. Despite its strength, we were unable to firmly placed this transition in the decay scheme. It does not connect any two of the previously known levels. The pure E0 character of the $259.9-\mathrm{keV}$ line implies that it connects two $0^{+}$states. The possibilitites that it feeds the ground state or de-excites the $721.2 \mathrm{keV}$ state, must be rejected, since a low lying $0^{+}$state would mean a dramatic departure from the systematics of the $0^{+}$ states in the region [19,20], and also: (even if weakly populated) it would have been likely found in the ${ }^{232} \mathrm{Th}\left(\mathrm{d},{ }^{6} \mathrm{Li}\right)^{228} \mathrm{Ra}$ reaction $[8,20]$. Furthermore, in the case of a $0^{+}$state at $461.3-\mathrm{keV}$, the intensity of I 6 would have to channel via an E0 transition to the ground state and/or via E2 397.4-keV transition to the $2_{1}^{+}$state. But no trace of either of these transitions has been found.

The most likely placement for the $259.9-\mathrm{keV}$ line is between the $721.2 \mathrm{keV} 0_{2}^{+}$state and a new $0^{+}$level at $981.1 \mathrm{keV}$. However, no $0_{3}^{+} \rightarrow 0_{1}^{+}$E0 transition of the energy of 981.1 $\mathrm{keV}$ has been found. The information on the $\mathrm{E} 0$ transitions which de-excite the $0_{3}^{+}$states, is very poor in the region. However, strong $\mathrm{E} 00_{3}^{+} \rightarrow 0_{2}^{+}$transitions do occur in vicinity of ${ }^{228} \mathrm{Ra}$. Such is the case of ${ }^{234} \mathrm{U}$, where the $0_{3}^{+}$state at 1044.5 is de-excited [21] to the $0_{2}^{+}$ 
state at $809.9 \mathrm{keV}$ via an intense E0 line of the energy of $234.6 \mathrm{keV}$, and to the $1_{1}^{-}$and $2_{1}^{+}$states via E1 and E2 transitions with the E0 / E1 / E2 intensity ratios of 10.4 / 8.6 / 100. However, no $0_{3}^{+} \rightarrow 0_{1}^{+} \mathrm{E} 0$ transition has been reported [21] in that nucleus.

For the postulated $0_{3}^{+}$state at $981.1 \mathrm{keV}$ in ${ }^{228} \mathrm{Ra}$, we observe no clear $\gamma$ ray at the energy of $\sim 507.0$ nor $\sim 917.3 \mathrm{keV}$ that could be associated with feeding of the $1_{1}^{-}$or $2_{1}^{+}$ states, respectively. Some trace of a $506.5(5) \mathrm{keV}$ line with the intensity of $\mathrm{I}_{506} \sim 1-2$ can be seen, and perhaps a weak $917.3 \mathrm{keV}$ line with a similar intensity exists in a cluster of weak lines at $\mathrm{E}_{\gamma} \sim 920 \mathrm{keV}$. For the latter, one estimates a limit of $\mathrm{I}_{917} \leq 2.5$. The absence of competing $\gamma$ radiation could mean that the rate of the $0_{3}^{+} \rightarrow 0_{2}^{+}$E0 transition is much higher in ${ }^{228} \mathrm{Ra}$ in comparison to ${ }^{234} \mathrm{U}$. The value of $\mathrm{X}_{321}$, defined as $\mathrm{X}_{321}=\mathrm{B}(\mathrm{E} 0$; $\left.0_{3}^{+} \rightarrow 0_{2}^{+}\right) / \mathrm{B}\left(\mathrm{E} 2 ; 0_{3}^{+} \rightarrow 2_{1}^{+}\right)$, which equals to $0.33(4)$ for ${ }^{234} \mathrm{U}$, would yield $\geq 11$ for ${ }^{228} \mathrm{Ra}$.

Further experimental studies are needed to clarify the placement of this transition. An aditional area of concern is the intensity balance for the $721.2 \mathrm{keV}$ level. In the adopted decay scheme [22] of ${ }^{228} \mathrm{Fr}$, the total feeding $\gamma$ intensity of $9.4(11)$ is matched by the deexcitation intensity of $8.0(5)$, thus leaving no room for an additional feeding of $\sim 6$ units that would be brought by the $259.9 \mathrm{keV}$ transition. However, the three $\gamma$ transitions assigned to feed this state, have been tentatively placed based on the energy match of the known levels and may belong elsewhere in the decay.

\subsection{Level half-life measurements}

Level lifetimes have been measured with the $\beta \gamma \gamma(t)$ method detailed in Refs. [23, $24,25]$. Two independent ${ }^{228} \mathrm{Ra}$ experiments were performed with the same experimental set up consisting of four detectors positioned around the beam deposition point in a rather close geometry. The timing information was obtained from time-delayed coincidences between the fast response $\beta$ - and $\gamma$-detectors, while coincidence with one of the two Ge detectors in the setup served to select the desired decay branch. A 3-mm thick NE111A plastic scintillator and a small (25 $\mathrm{mm}$ in height) $\mathrm{BaF}_{2}$ crystal served as the fast timing $\beta$ and $\gamma$ detectors, respectively. As Ge detectors we used a Low-Energy-Photon (LEP) and High-Purity-Ge (HPGe) detectors in the first run and two HPGe detectors in the second one.

A multi-parameter time-delayed coincidence system was set between the $\beta$ detector and any combination of the $\gamma$ detectors. The fast timing analysis was performed on the double coincidence $\beta \gamma(\mathrm{t})$ events involving the fast response $\beta-\mathrm{BaF}_{2}$ detectors and the triple coincidence $\beta \gamma \gamma(\mathrm{t})$ events recorded with the $\beta-\mathrm{BaF}_{2}-\mathrm{Ge}$ detectors. The exact composition of the $\mathrm{BaF}_{2}$ energy gates, which was needed for the analysis of the $\beta-\mathrm{BaF}_{2}$ and $\beta-\mathrm{BaF}_{2}-\mathrm{Ge}$ data, was provided by the $\beta-\mathrm{Ge}$ and $\beta-\mathrm{Ge}-\mathrm{Ge}$ coincidences which were collected at the same time. Using the latter data, we had identified the $\gamma$ transitions observed in the run (no major impurity lines were observed) and confirmed that the placement of intense transitions is in agreement with the decay scheme of ${ }^{228} \mathrm{Fr}$ proposed by Ruchowska et al. [7]. We have also searched, but with no success, for level lifetimes in the range of $100 \mathrm{~ns}<\mathrm{T}_{1 / 2}<2 \mu$ s using the $\beta \gamma(\mathrm{t})$ coincidences recorded with the LEP detector.

\subsubsection{Centroid-shift $\beta \gamma \gamma(t)$ measurements}

In the centroid shift measurement for direct $\beta$ feeding of a level of interest, the mean life of a level $\left(\tau=\mathrm{T}_{1 / 2} / \ln 2\right)$ is given simply by the difference between the centroid of the delayed time spectrum and the prompt centroid of the same $\mathrm{E}_{\gamma}$. For the indirect feeding where the level of interest is fed by a $\gamma$ ray from a higher level, the mean life of interest is the difference between the centroid shift of the spectrum gated by the de-exciting $\gamma$ 
ray and the centroid shift of the spectrum gated by the feeding $\gamma$ transition. The latter serves here as a reference point. The prompt points, as well as the reference points, are internal to the decay and serve to renormalize the calibration prompt curves for the $\mathrm{BaF}_{2}$ detector that are measured off-line. For the lifetimes of the $1_{1}^{-}$and $3_{1}^{-}$states listed in table 2 , the $\gamma$ transitions feeding the state of interest provide the time reference points, while the transitions de-exciting these states provide the time-delayed shift. However, important corrections must be applied before this shift can be converted into the mean life of a level of interest.

In comparison to the measurements in ${ }^{227} \mathrm{Fr}$ or ${ }^{227} \mathrm{Ra}$ discussed in refs. [1, 2] (see for example fig. 5 in ref. [2]), the present case is somewhat different. In odd-A ${ }^{227} \mathrm{Fr}$ and ${ }^{227} \mathrm{Ra}$ there are few feeding transitions. Some of them are dominant and well separated in the coincident $\mathrm{BaF}_{2}$ spectra as can be seen in fig. 2 in ref. [2]. Consequently, it was possible to select for the timing analysis only the time spectra gated on the full energy peaks in $\mathrm{BaF}_{2}$ for the time-delayed and reference points measured on-line, and also for the prompt points measured off-line. The procedures were thus much simplified.

In contrast, the $\mathrm{BaF}_{2}$ spectra coincident with the transitions de-exciting the $4_{1}^{+}, 1_{1}^{-}$ and $3_{1}^{-}$states in ${ }^{228} \mathrm{Ra}$ (selected in the Ge detector) are flat and featureless as they are formed from an overlap of a large number of rather weak transitions feeding the states from above. Furthermore, centroids of the time spectra gated on the prompt Compton continuum are systematically shifted by a few picoseconds from those gated on the full energy peaks (see for example fig. 4 in ref. [25]). That difference, which is critical for the present study, has been measured precisely and corrected for with the help of the calibration curves.

Table 2: Half-lives of the excited states in ${ }^{228}$ Ra.

\begin{tabular}{cccc}
\hline \hline Level & $\mathbf{J}^{\pi}$ & $\begin{array}{c}T_{1 / 2} \\
\text { previous work }^{a)} \\
(\mathrm{ps})\end{array}$ & $\begin{array}{c}T_{1 / 2} \\
\text { present work } \\
(\mathrm{ps})\end{array}$ \\
\hline 63.8 & $2_{1}^{+}$ & $550(40)$ & $550(20)$ \\
204.7 & $4_{1}^{+}$ & & $181(3)$ \\
474.1 & $1_{1}^{-}$ & & $\left.\leq 7^{b}\right)$ \\
537.6 & $3_{1}^{-}$ & & $\leq 6^{b}$ \\
\hline \hline
\end{tabular}

1) From ref. [10].

2) $2 \sigma$ limit.

The most important aspect of the $\beta \gamma \gamma(\mathrm{t})$ method used here is the ability to provide three calibration curves that characterize the $\mathrm{BaF}_{2}$ detector: the prompt curve, which gives the time response of the $\mathrm{BaF}_{2}$ detector as a function of the $\gamma$-ray energy for the fullenergy peaks (FEP), the time response curve for the complete energy spectrum (Compton plus FEP) due to a monoenergetic $\gamma$ transition of a medium energy (usually selected with $E_{\gamma} \sim 1.5 \mathrm{MeV}$ ), and the Compton-correction curve, which describes the time shift between Compton events of the same energy but due to prompt primary $\gamma$ rays of different energy. These curves are essential for the centroid shift measurements.

The first two curves, which are the most critical in the present analysis, were obtained off-line at ISOLDE. In particular, the prompt curve was measured off-line using the pre-calibrated sources of ${ }^{154} \mathrm{Eu} \rightarrow{ }^{154} \mathrm{Gd}$ in the first experiment and ${ }^{140} \mathrm{Ba} \rightarrow{ }^{140} \mathrm{La} \rightarrow{ }^{140} \mathrm{Ce}$ 


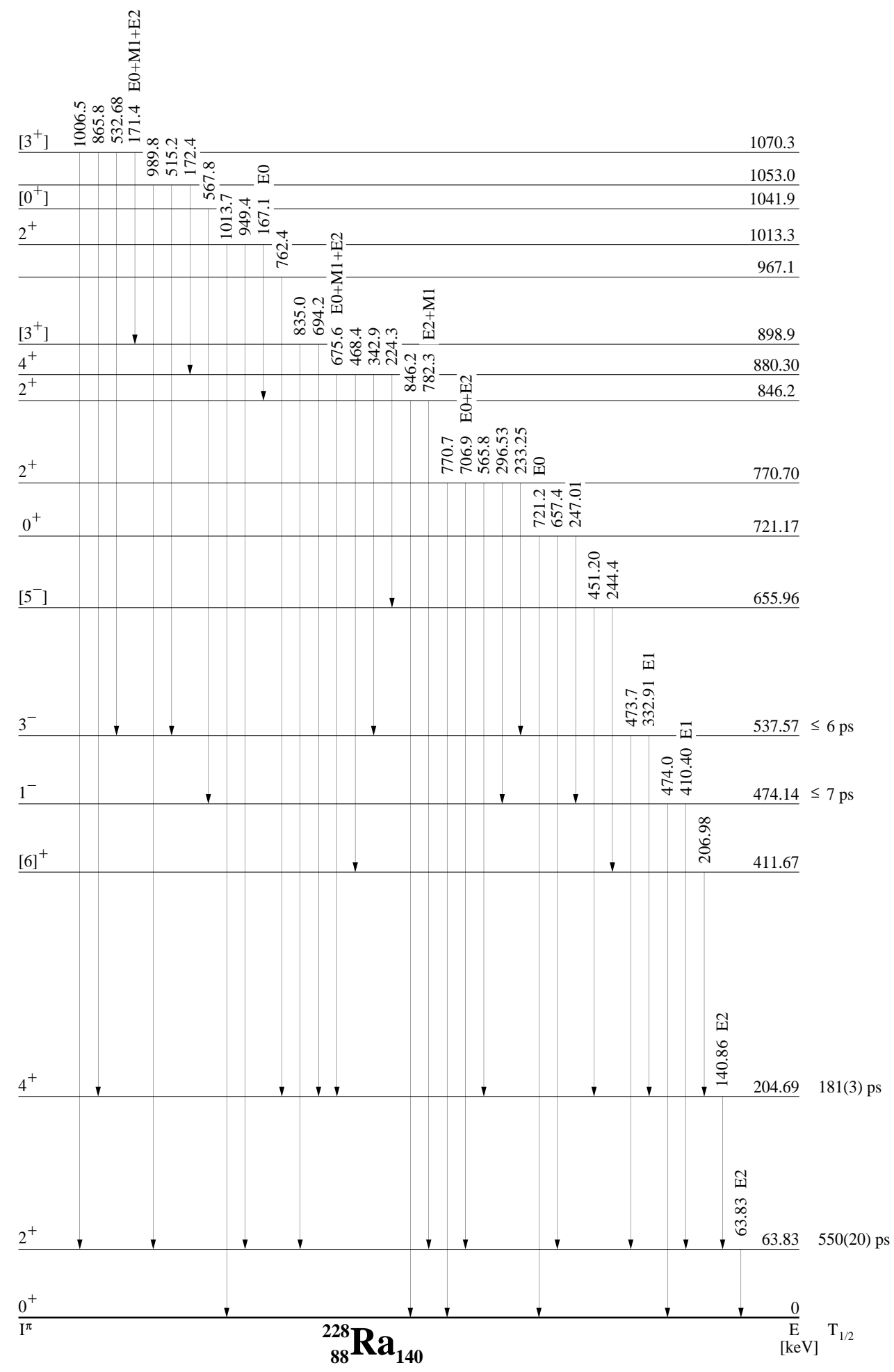

Figure 2: Partial level scheme for the decay of ${ }^{228} \mathrm{Fr} \rightarrow{ }^{228} \mathrm{Ra}$. Data are from ref. [7] except for the multipolarities, spin/parity assignments and level lifetimes, which are from this work. 
in the second experiment, that were prepared at the OSIRIS mass separator at Studsvik $[26,27]$. More detailed time calibration of the full energy peak and the Compton-correction curves were done at OSIRIS using a variety of beams while maintaining the experimental geometry as close as possible to that previously used at ISOLDE. Some details of the method including corrections applied are given in [23, 25].

For ${ }^{228} \mathrm{Ra}$, the reference points were obtained by gating on the higher energy part of the Compton continuum in the $\mathrm{BaF}_{2}$ spectra coincident with the 140, 332, 410, and $473 / 474 \mathrm{keV}$ transitions selected in the Ge detector (see fig. 2). As expected these individual data sets overlapped quite closely since the lifetimes of the high-lying levels at the excitation energy of 2-3 MeV that are involved here, are predicted to be in the femtosecond regime making all these data points basically prompt. By correcting for a small shift between the Compton and full energy peak positions (via the calibration curves), these points became the reference points for the measurements discussed next.

The half-life limit for the $1_{1}^{-}$state at $474.2 \mathrm{keV}$ (which is de-excited by the 410 $\mathrm{keV}$ and $474 \mathrm{keV}$ transitions) was obtained by averaging four independent results: four shifts from the reference points of the time-delayed spectra selected with a common $\mathrm{BaF}_{2}$ gate on the unresolved $410 / 474 \mathrm{keV}$ peak, a common gate on the $\beta$ spectrum, and the individual gates in Ge on the $296,567,613$, and $1567 \mathrm{keV}$ transitions, respectively, which feed the $474.2 \mathrm{keV}$ level directly from above. In the case of the $3_{1}^{-}$level at $537.6 \mathrm{keV}$, the half-life limit represents an average of ten time-delayed results. For every one of the five Ge peaks selected at the energies of 233, 343, 550, 645 and $1572 \mathrm{keV}$ (which are the strongest transitions directly feeding the $537.6 \mathrm{keV}$ level) one could select in the coincident $\mathrm{BaF}_{2}$ spectra separately the full energy peaks of the $332-$ and $473-\mathrm{keV}$ transitions deexciting the level. The individual results for the $474.2-$ and $537.6-\mathrm{keV}$ levels had common characteristics: the centroid positions of the time-delayed spectra consistently overlaped with the curve defined by the reference points, thus yielding only limits of the level halflives. The final values quoted in table 2 , as $\mathrm{T}_{1 / 2} \leq 7 \mathrm{ps}$ and $\leq 6 \mathrm{ps}$ for the $1_{1}^{-}$and $3_{1}^{-}$ states, respectively, represent $2 \sigma$ limits and were obtained by averaging results from two independent series of measurements.

\subsubsection{Lifetimes obtained from shape de-convolution}

In the shape de-convolution method, lifetimes with $T_{1 / 2} \geq 100 \mathrm{ps}$ are obtained from the asymmetry or slope on the "delayed" side of the time spectrum by fitting the spectrum to an analytical function with the prompt response approximated by a Gaussian. A detailed analysis of the fast timing $\beta \gamma(\mathrm{t})$ and $\beta \gamma \gamma(\mathrm{t})$ coincidences has revealed no trace of any lifetimes measurable by the shape de-convolution or slope-fitting with the exception of the 63.8 and $204.7 \mathrm{keV}$ levels. The half-lives for these states listed in table 2 are from the $\beta \gamma(\mathrm{t})$ measurements characterized by higher statistics. These results are in a full agreement with those from the $\beta \gamma \gamma(\mathrm{t})$ analysis. A simplified description of the analysis process is given below.

In the analysis of the $\beta \gamma(t)$ coincidences a series of sortings was performed with a variety of gating conditions in order to check the conditions of the experiment and to find optimal gates (e.g.: on the $\beta$ spectrum). Then a common gate was set on the $\beta$ spectrum and a series of gates were selected on the delayed side of the time spectrum (projected from all data) outside of the prompt region. The data was sorted onto the $\mathrm{BaF}_{2}$ energy. Figures $3 \mathrm{a}$ and $3 \mathrm{c}$ show the $\mathrm{BaF}_{2}$ energy spectra gated by those $\gamma$ events that were recorded from 0.4 to $1.4 \mathrm{~ns}$ and from 1.6 to $5.0 \mathrm{~ns}$, respectively, after the $\beta$ decay of ${ }^{228} \mathrm{Fr}$. The coincident energy spectra reveal various $\gamma$ components contributing to 

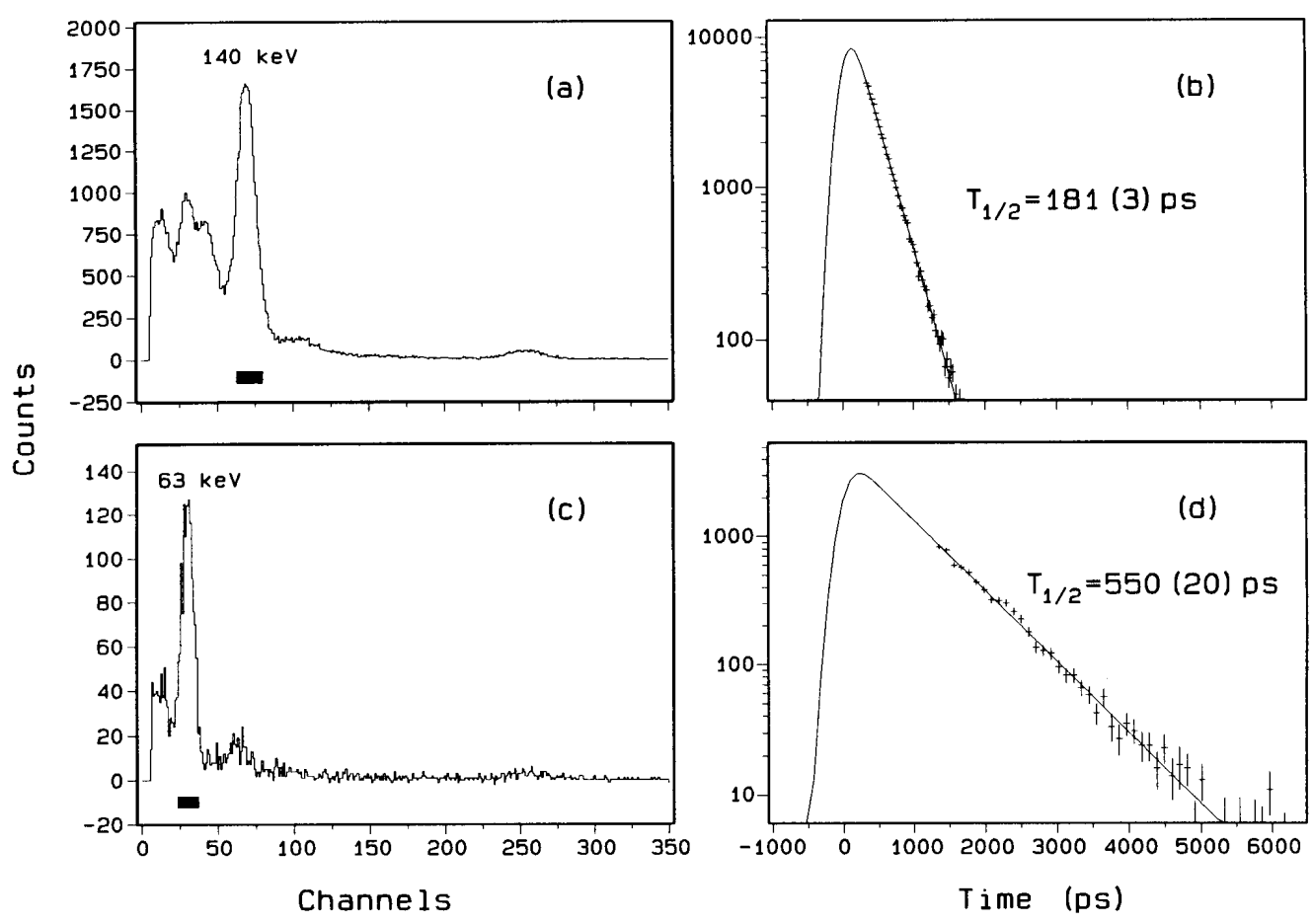

Figure 3: (a) and (c): $\mathrm{BaF}_{2}$ energy spectra sorted from the $\beta \gamma(\mathrm{t})$ coincidences by selecting those $\gamma$ events which were recorded in $\mathrm{BaF}_{2}$ from 0.4 to $1.4 \mathrm{~ns}$ (panel (a)) and from 1.6 to $5.0 \mathrm{~ns}$ (panel (c)) after the $\beta$ decay of ${ }^{228} \mathrm{Fr}$. The contribution from randoms and slower time components has been subtracted. (b): Coincident $\beta \gamma(\mathrm{t})$ time spectrum gated by the $140 \mathrm{keV} \gamma$ transition selected in $\mathrm{BaF}_{2}$ (marked by a black rectangle in panel (a)) and by $\beta$ particles. Fitting of the time spectrum is limited to the time range from 0.4 to $1.4 \mathrm{~ns}$. (d): Similar to (b) but for a coincident time spectrum gated by the $63 \mathrm{keV}$ transition (marked by a black rectangle in panel (c)). Fitting of the time spectrum is limited to the time range from 1.6 to $5.0 \mathrm{~ns}$. See text for a full discussion.

the asymmetry of the time spectrum. A small contribution comes from the usual sources: the backscattered radiation $\left(\mathrm{E}_{\gamma} \sim 220 \mathrm{keV}\right)$ and the $511 \mathrm{keV} e^{-} e^{+}$annihilation radiation. The main contribution, however, comes from two $\gamma$ transitions of energy 140.9 and 63.8 $\mathrm{keV}$ de-exciting states with half-lives longer than $100 \mathrm{ps}$. Thus one can see a strong and well separated peak at $140 \mathrm{keV}$ in fig. $3 \mathrm{a}$ and a $63 \mathrm{keV}$ peak in fig. $3 \mathrm{c}$. The peak at about $90 \mathrm{keV}$ (channel $\sim 43$ in fig. $3 \mathrm{a}$ ) is due to the Ra X-rays associated with the conversion electron process for the $140 \mathrm{keV}$ transition.

In the final step, gates were selected on the 140 and $63 \mathrm{keV}$ peaks in the $\mathrm{BaF}_{2}$ spectrum (black rectangles in figs. $3 \mathrm{a}$ and $3 \mathrm{c}$ ) and, with a common gate on the $\beta$ spectrum, the data was sorted back onto the fast timing spectra. The resultant time spectra for the $140 \mathrm{keV}$ and $63 \mathrm{keV}$ transitions are shown in figs. $3 \mathrm{~b}$ and $3 \mathrm{~d}$. The shape fitting of the time spectra outside of the prompt region gives $\mathrm{T}_{1 / 2}=550(20)$ ps for the $2_{1}^{+}$level and $181(3) \mathrm{ps}$ for the $4_{1}^{+}$state. The first result is in very good agreement with the value $\mathrm{T}_{1 / 2}$ $=550(40)$ ps reported in ref. [10]. 


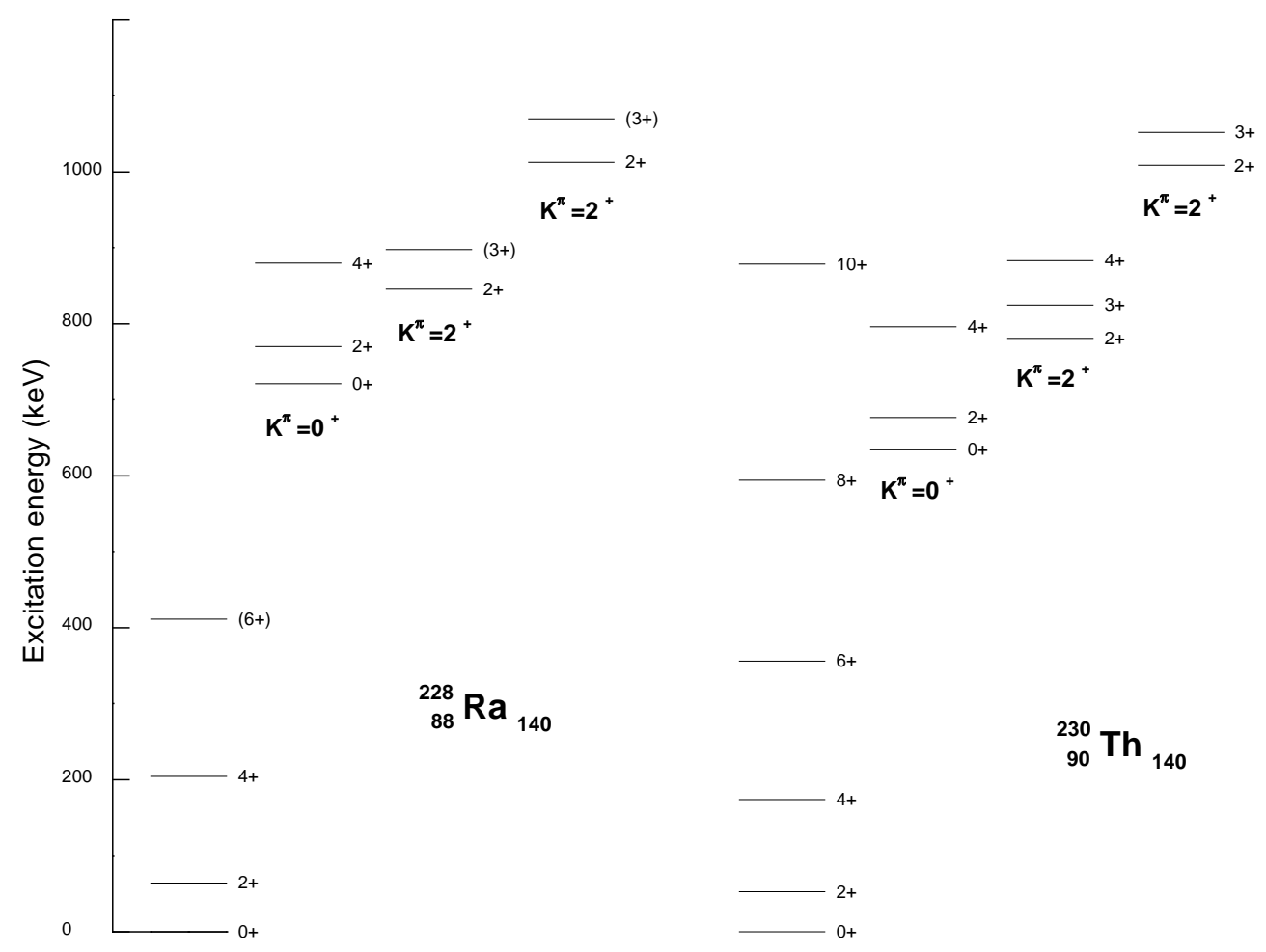

Figure 4: Comparison of band structures in ${ }^{228} \mathrm{Ra}$ (this work) and ${ }^{230} \mathrm{Th}$ (ref. [31]).

\section{Discussion of the results}

\subsection{Level scheme}

The interpretation of the even parity states of ${ }^{228} \mathrm{Ra}$ is presented in fig. 4 alongside with a partial level scheme of its isotone ${ }^{230} \mathrm{Th}$. The conversion electron as well as the fast timing data discussed in the next subsection, confirm the spin values which were tentatively assigned by Ruchowska et al. [7].

\subsection{1 $\mathrm{K}^{\pi}=0^{+}$ground-state band}

The assignments of $\mathrm{I}^{\pi}=2^{+}$and $4^{+}$to the 63.8 and $204.7 \mathrm{keV}$ levels, respectively, are based on the E2 multipolarities of the 63.8 and $140.9 \mathrm{keV}$ transitions. The weakly populated $411.7 \mathrm{keV}$ state appears to be the next member of the rotational ground state band, but there is still no direct experimental evidence for its $6^{+}$assignment.

\subsubsection{First excited $K^{\pi}=0^{+}$band}

The $\mathrm{K}=0$ assignment for the $721.2,770.7$ and $880.3 \mathrm{keV}$ levels is firmly established either by a pure E0 transition or by a strong $\mathrm{E} 0$ contribution to the $\mathrm{E} 0+\mathrm{M} 1+\mathrm{E} 2$ transitions (see table 1 ) to the $0^{+}, 2^{+}$and $4^{+}$levels of the ground-state band. The values of $\mathrm{X}$, a dimensionless ratio of the E0- and E2-transition probabilities [28] that gives a measure of the E0 decay rate, have been evaluated for the $721.2,706.9$ and $675.6 \mathrm{keV}$ E0 transitions as $0.07(2), 0.11(1)$ and $0.20(8)$, respectively. Unfortunately, the experimental $X$ values cannot discriminate between predictions of two very different theoretical models: $\mathrm{X}_{\beta}=0.19$ is predicted in the case of beta vibration [29] (we take $\mathrm{X}_{\beta}=4 * \beta_{0}^{2}$ and the quadrupole deformation parameter $\beta_{0}=0.22$ ) and $\mathrm{X} \sim 0.25$ for the cluster model $-\mathrm{a}$ prediction approximately constant for a different numbers of bosons [30]. 


\subsection{3 $\mathrm{K}^{\pi}=2^{+}$bands}

Two excited $\mathrm{K}^{\pi}=2^{+}$bands, with bandheads at 846.2 and $1013.3 \mathrm{keV}$, connected by rather strong $167.1 \mathrm{keV}$ E0 transition, were proposed by Ruchowska et al. [7]. In the present work a positive parity has been established for the $846.2 \mathrm{keV}$ level, interpreted as a $\gamma$-vibrational state. The present data established an E0 component in the $171.4 \mathrm{keV}$ transition connecting the proposed $\mathrm{I}^{\pi}=3^{+}$members of the $\mathrm{K}^{\pi}=2^{+}$bands. The level at $1013.3 \mathrm{keV}$ is assigned as an $\mathrm{I}^{\pi} \mathrm{K}=2^{+} 2$ state by analogy with levels in the isotone ${ }^{230} \mathrm{Th}$ [31], and extends the systematics of the second $\mathrm{K}^{\pi}=2^{+}$band observed in heavier actinide nuclei [32]. The nature of the second low-lying collective $\mathrm{K}^{\pi}=2^{+}$bands is not very clear. Their excitation energy is much lower than predicted for the two-phonon $\beta+\gamma$ state in the simple model of harmonic nuclear vibrations. Clearly, the second low-lying $\mathrm{K}^{\pi}=2^{+}$ bands need further study.

\subsection{4 $K^{\pi}=0^{-}$band}

The E1 multipolarities for the 332.9 and $410.4 \mathrm{keV}$ transitions (see table 1) provide firm spin and negative parity assignments of $\mathrm{I}^{\pi}=1^{-}$and $3^{-}$for the 474.1 and $537.6 \mathrm{keV}$ levels, respectively, and thus confirm the previous tentative assignments given to these states [7]. However, the assignment of $\mathrm{I}^{\pi}=5^{-}$for the $656.0 \mathrm{keV}$ level remains tentative.

\subsection{Transition probabilities}

A summary of the measured transition rates is presented in tables 3 and 4 . The first two columns list the energies of the connecting $\gamma$ transitions and the spin/parities of the initial and final states. The reduced transition probabilities, $\mathrm{B}\left(\mathrm{XL} ; \mathrm{I}_{i} \rightarrow \mathrm{I}_{f}\right)$, are given in column three in absolute units and in column four in Weisskopf units. The last column lists the absolute value of the quadrupole moment, $Q_{0}$ :

$$
B\left(E 2 ; I_{i} \rightarrow I_{f}\right)=\frac{5}{16 \pi} Q_{0}^{2}\left\langle I_{i} K_{i} 20 \mid I_{f} K_{f}\right\rangle^{2},
$$

or the electric dipole moment, $D_{0}$ :

$$
B\left(E 1 ; I_{i} \rightarrow I_{f}\right)=\frac{3}{4 \pi} D_{0}^{2}\left\langle I_{i} K_{i} 10 \mid I_{f} K_{f}\right\rangle^{2},
$$

derived using the rotational formulas. Although these formulas are strictly applicable to rigid rotors, nevertheless, they are widely used to provide consistent means of intercomparison of the $\mathrm{B}(\mathrm{E} 2)$ or $\mathrm{B}(\mathrm{E} 1)$ strength between even-even and odd-A nuclei as well as for the transitions de-exciting various band members.

\subsubsection{Quadrupole collectivity}

${ }^{228} \mathrm{Ra}$ is located in a quadrupole transitional region but close to the strongly deformed nuclei. This is confirmed by the ratio of energies of the $4_{1}^{+}$to the $2_{1}^{+}$states, $\mathrm{R}=$ $E_{4}^{+} / E_{2}^{+}$, which is equal to 3.21 - a value close to the rigid rotor limit of 3.33 . The new results of this study provide a further confirmation of the proximity of ${ }^{228} \mathrm{Ra}$ to this limit. One feature of a rigid rotor is a constancy of the quadrupole moment for members of its rotational band. As seen in table 3, the quadrupole moments measured for the two lowest members of the $\mathrm{K}=0^{+}$band are, indeed, equal to within the precision of the measurement.

As mentioned in the Introduction, measurements of the isotope shifts in both the atomic and ionic resonance lines have been performed on the radium isotopes in the mass range $208 \leq \mathrm{A} \leq 232$ using the on-line collinear fast-beam laser spectroscopy technique 
Table 3: E2 transition rates in ${ }^{228}$ Ra.

\begin{tabular}{|c|c|c|c|c|}
\hline $\begin{array}{c}\mathrm{E}_{\gamma} \\
(\mathrm{keV})\end{array}$ & $\overline{\mathrm{I}_{i}^{\pi} \rightarrow \mathrm{I}_{f}^{\pi}}$ & $\begin{array}{c}\left.\mathrm{B}(\mathrm{E} 2)^{a}\right) \\
\left(\mathrm{e}^{2} \mathrm{fm}^{4}\right)\end{array}$ & $\begin{array}{c}\mathrm{B}(\mathrm{E} 2)^{b)} \\
\text { (W.u.) }\end{array}$ & $\begin{array}{c}\left|\mathrm{Q}_{0}\right| \\
\left(\mathrm{e} \cdot \mathrm{fm}^{2}\right) \\
\end{array}$ \\
\hline 63.8 & $2_{1}^{+} \rightarrow 0_{1}^{+}$ & $11770(490)$ & $142(6)$ & $769(16)$ \\
\hline 140.9 & $4_{1}^{+} \rightarrow 2_{1}^{+}$ & $17160(370)$ & $207(4)$ & $776(8)$ \\
\hline
\end{tabular}

[9]. In their study, where the laser-spectroscopic results have been examined in terms of evidence for octupole instability, Ahmad et al. [9] compared the deformed part $\delta\left\langle r^{2}\right\rangle_{d e f}^{228, A}$ deduced from the measured isotope shifts and representing the change in $\left\langle\beta^{2}\right\rangle=\sum_{i}\left\langle\beta_{i}^{2}\right\rangle$ (with $i=2,3,4 \ldots$ ), with droplet model calculations that accounted only for the even multipole deformations $(i=2,4)$. The droplet calculations included a $\beta_{2}$ term deduced from the experimental $\mathrm{B}\left(\mathrm{E} 2 ; 2_{1}^{+} \rightarrow 0_{1}^{+}\right)$rates for the even-even nuclei and a $\beta_{4}$ term, which represented theoretical correction. The discrepancies between these two data sets, which are particularly large below ${ }^{226} \mathrm{Ra}$ (see fig. 5 in ref. [9]), can be eliminated by the inclusion of the octupole mode with $\beta_{3}$ values of the order predicted by theory. For example, a gap between data points for ${ }^{224} \mathrm{Ra}$ requires $[9]\left\langle\beta_{3}^{2}\right\rangle^{1 / 2}=0.07$ in agreement with theoretical predictions.

However, the comparison presented in ref. [9] had to be viewed with caution largely necessitated by the status of the experimental $\mathrm{B}\left(\mathrm{E} 2 ; 2_{1}^{+} \rightarrow 0_{1}^{+}\right)$results for ${ }^{222} \mathrm{Ra}$ and ${ }^{228} \mathrm{Ra}$. In particular, a strong enhancement of the $\mathrm{B}(\mathrm{E} 2)$ rate, which has been measured [10] for ${ }^{222} \mathrm{Ra}$ and recognized in ref. [33] as an important sign of a mixing of a deformed cluster state in the ground state of ${ }^{222} \mathrm{Ra}$, has been taken in ref. [9] as doubtful. Consequently, Ahmad et al. disregarded the data point at ${ }^{222} \mathrm{Ra}$. On the other hand, the result for ${ }^{228} \mathrm{Ra}$ which provided a crucial renormalization point between both data sets [9], suffered from a large statistical uncertainty making it difficult to draw any firm conclusion on the size of the $\beta_{3}$ contribution. As for ${ }^{222} \mathrm{Ra}$, our direct lifetime measurement [34] performed recently, contradicts the previous measurement on this nucleus and validates the suspicion of Ahmad et al.

The new half-life result for the $2_{1}^{+}$state in ${ }^{228} \mathrm{Ra}$ has a numerical value identical to the previous one, but with improved precision, see table 2 . The two measurements can be combined to an average value of $\mathrm{T}_{1 / 2}=550(18) \mathrm{ps}$. It gives an identical $\left.<\beta_{2}^{2}\right\rangle^{1 / 2}$ as the one used before, see table 3 and fig. 5 in ref. [9], but with about $1 / 2$ of the previous uncertainty. Consequently, the new result reinforces the conclusions of Ahmad et al. , that there exists a sizeable discrepancy between the two data sets. Furthermore, it is more clear now, that the size of the discrepancies and the position of its maximum are consistent with the predictions of octupole deformation.

Note, that Ahmad et al. [9] assumed a negligible $\beta_{3}$ for ${ }^{228} \mathrm{Ra}$ and thus used the nucleus to renormalize the isotope shift and the droplet calculation results. In the next section we examine the arguments for the octupole collective features in ${ }^{228} \mathrm{Ra}$ which imply a sizable dynamical $\beta_{3}$ in this nucleus. Thus, it would be more proper to use a heavier $\mathrm{Ra}$ nucleus as a normalization point. One candidate is ${ }^{230} \mathrm{Ra}$ on which the fast timing measurements have been recently performed [27]. 


\subsection{2 $B($ E1) rates}

As a result of this study, the $\mathrm{B}(\mathrm{E} 1)$ rates have been measured for the first time in ${ }^{228} \mathrm{Ra}$. The lower limits for the $\mathrm{B}(\mathrm{E} 1)$ rates, which range from 2.9 to $5.2 \times 10^{-4} \mathrm{e}^{2} \mathrm{fm}^{2}$, are surpisingly fast, giving $\left|D_{0}\right| \geq 0.07$ e.fm, and clearly reveal the presence of sizeable octupole correlations in this nucleus. It is now possible to examine the theoretical predictions for ${ }^{228} \mathrm{Ra}$.

The microscopic calculations based on the constrained Hartree-Fock plus BCS theory as well as on the adiabatic time-dependent Hartree-Fock in the cranking approximation with Gogny force have been performed by Egido and Robledo [4] in the case of ${ }^{218-230} \mathrm{Ra}$. These calculations predict fast $\mathrm{E} 1$ rates for ${ }^{228} \mathrm{Ra}$ with $\mathrm{B}(\mathrm{E} 1) \sim 7 \times 10^{-3} \mathrm{e}^{2} \mathrm{fm}^{2}$, driven by the octupole vibrational mode of excitation. The same authors also performed a microscopic parity-projection calculation for ${ }^{218-230} \mathrm{Ra}$ using the realistic interaction of Gogny [5] and obtained practically the same $\mathrm{B}(\mathrm{E} 1)$ rates with $\mathrm{B}(\mathrm{E} 1)=3 \times 10^{-3} \mathrm{e}^{2} \mathrm{fm}^{2}$ predicted for ${ }^{228} \mathrm{Ra}$.

The calculations by Denisov [11] predict a sizable octupole deformation in ${ }^{226} \mathrm{Ra}$ with $\beta_{3}=0.09$, a much smaller one in ${ }^{228} \mathrm{Ra}, \beta_{3}=0.02$, and a reflection symmetry for ${ }^{230} \mathrm{Ra}$. Consequently, he predicts a dramatic drop in the $\mathrm{B}(\mathrm{E} 1)$ strength for the Ra nuclei between $\mathrm{N}=138$ and 142 with $D_{0}=0.27 \mathrm{e} \cdot \mathrm{fm}$ and $0.066 \mathrm{e} \cdot \mathrm{fm}$ for ${ }^{226} \mathrm{Ra}$ and ${ }^{228} \mathrm{Ra}$, respectively, and zero for ${ }^{230} \mathrm{Ra}$. For ${ }^{228} \mathrm{Ra}$ this implies $\mathrm{B}(\mathrm{E} 1) \approx 4 \times 10^{-4} \mathrm{e}^{2} \mathrm{fm}^{2}$ which is close to the measured limits. One should note, however, that the calculations by Denisov correlate E1 strength basically with the size of the reflection asymmetry and thus they do not account for the octupole vibrations nor do they predict the well known quenching of the $\mathrm{B}(\mathrm{E} 1)$ strength in ${ }^{224} \mathrm{Ra}$.

Table 4: E1 transition rates in ${ }^{228}$ Ra.

\begin{tabular}{ccccc}
\hline \hline $\begin{array}{c}\mathrm{E}_{\gamma} \\
(\mathrm{keV})\end{array}$ & $\mathrm{I}_{i}^{\pi} \rightarrow \mathrm{I}_{f}^{\pi}$ & $\begin{array}{c}\left.\mathrm{B}(\mathrm{E} 1)^{a}\right) \\
\left(\mathrm{e}^{2} \mathrm{fm}^{2}\right)\end{array}$ & $\begin{array}{c}\mathrm{B}(\mathrm{E} 1)^{b)} \\
(\text { W.u. })\end{array}$ & $\begin{array}{c}\left|\mathrm{D}_{0}\right| \\
(\mathrm{e} \cdot \mathrm{fm})\end{array}$ \\
\hline 332.9 & $3_{1}^{-} \rightarrow 4_{1}^{+}$ & $\geq 3.5 \times 10^{-4}$ & $\geq 1.5 \times 10^{-4}$ & $\geq 0.051$ \\
410.4 & $1_{1}^{-} \rightarrow 2_{1}^{+}$ & $\geq 3.6 \times 10^{-4}$ & $\geq 1.5 \times 10^{-4}$ & $\geq 0.047$ \\
473.7 & $3_{1}^{-} \rightarrow 2_{1}^{+}$ & $\geq 5.2 \times 10^{-4}$ & $\geq 2.2 \times 10^{-4}$ & $\geq 0.071$ \\
474.0 & $1_{1}^{-} \rightarrow 0_{1}^{+}$ & $\geq 2.9 \times 10^{-4}$ & $\geq 1.2 \times 10^{-4}$ & $\geq 0.060$ \\
\hline \hline
\end{tabular}

5) Using $\mathrm{T}_{1 / 2}$ from table 2 and total conversion coefficients, $\alpha_{T}$,

from ref. [18]. The uncertainty in $\alpha_{T}$ is taken as $\pm 2 \%$.

6) $\mathrm{B}(\mathrm{E} 1)_{W}=2.406 \mathrm{e}^{2} \mathrm{fm}^{2}$.

The schematic model calculation of Butler and Nazarewicz [12] predicts dipole moments in the reflection-asymmetric nuclei within the shell correction method. For the even nuclei of $\mathrm{Ra}$ and $\mathrm{Th}$, the authors use the theoretical equilibrium deformations $\beta_{2}-\beta_{8}$ from the calculation of Sobiczewski et al. [13] based on the Nilsson-Strutinsky approach with folded-Yukawa and Woods-Saxon deformed potentials. The latter calculation predicts disappearance of the octupole barrier height $\left(E_{o m}\right)$ for Ra at $\mathrm{N} \geq 140$ and for Th at $\mathrm{N} \geq 138$ where it gives $\beta_{\text {odd }}=0$. These cases represent reflection symmetric nuclei, for which the schematic model can provide no prediction, since it is not valid for octupole vibrational model. However, it is an open question whether ${ }^{228} \mathrm{Ra}$, located at the border line, is in fact reflection symmetric. Various calculations give a different estimate of $\left|\beta_{3}\right|$ for ${ }^{228} \mathrm{Ra}$. For example, its value is zero in ref. [13], 0.02 in ref. [11], or 0.058 in ref. [6]. 
Note, that the $\beta_{3}$ parameter gives only a partial picture. Another important parameter is the octupole barrier height [13] $\left(E_{o m}\right)$, which is usually quite small for the Ra nuclei near $N=140$. It means that the ${ }^{226-230}$ Ra nuclei exhibit basically a vibrational mode of collective octupole excitations, and thus one would expect a decrease in the E1 rates to be very gradual in similarity to the predictions made by Egido and Robledo [4, 5]. This would be in contrast to the schematic model predictions [12] or the calculation by Denisov [11], which do not include a proper treatment of octupole vibrations, and thus predict a sharp drop in the E1 rates related to the decrease in the value of $\beta_{3}$ along the transition to reflection symmetry.

The $\mathrm{B}(\mathrm{E} 1)$ rates measured in this work provide clear evidence that there is no sharp drop in the $\mathrm{B}(\mathrm{E} 1)$ rates between ${ }^{227} \mathrm{Ra}$ and ${ }^{228} \mathrm{Ra}$. The possibility is left open for the heavier nuclei of ${ }^{229} \mathrm{Ra}$ and ${ }^{230} \mathrm{Ra}$, which are the subject of our next investigation. However, the current results do not provide a clear indication of which theoretical approach is correct in the case of ${ }^{228} \mathrm{Ra}$. As demonstrated by Denisov, even a modest reflection asymmetry may yield the $\mathrm{B}(\mathrm{E} 1)$ rates consistent with the measurement.

The present measurement disproved the previous estimate of $D_{0}=0.011(1) \mathrm{e} \cdot \mathrm{fm}$ quoted in ref. [12] for ${ }^{228} \mathrm{Ra}$ (see the Introduction for details). If such a low value would be correct, it would present a challenge to our understanding of the octupole transitional region and in particular to the calculations of Egido and Robledo [4, 5].

\section{Summary}

In the present study we have performed conversion electron measurements with a mini-orange $\beta$ spectrometer, which have clarified the assignment of spins and parities to the collective states in ${ }^{228} \mathrm{Ra}$. Strong $\mathrm{E} 0$ transitions have been identified as connecting the $0^{+}, 2^{+}$and $4^{+}$members of the first excited $\mathrm{K}^{\pi}=0^{+}$band with the respective members of the ground state band. The present study established an E0 component in the 171.4 $\mathrm{keV}$ transition connecting the proposed $\mathrm{I}^{\pi}=3^{+}$members of the $\mathrm{K}^{\pi}=2^{+}$bands. However, further studies are needed to clarify the position of the intense $259.9 \mathrm{keV}$ E0 transition.

Our fast-timing $\beta \gamma \gamma(t)$ measurements have revealed lifetimes of $\mathrm{T}_{1 / 2}=550(20) \mathrm{ps}$ and 181(3) ps for the $2_{1}^{+}$and $4_{1}^{+}$members of the $\mathrm{K}=0^{+}$band. The first result confirms the previous measurement [10] but with much improved precision. The quadrupole moments deduced for the first two members of the ground state band were found equal within the statistical precision, as expected for a rigid rotor. Furthermore, the new results allow a re-examination of the analysis of Ahmad et al. [9] and significally strengthen his argument for a $\beta_{3}$ contribution to the deformed part $\delta\left\langle r^{2}\right\rangle_{d e f}^{228, A}$ deduced from the measured isotope shifts.

The fast timing study yields the first measurement of $\mathrm{B}(\mathrm{E} 1)$ rates in ${ }^{228} \mathrm{Ra}$. The half-life limits of $\mathrm{T}_{1 / 2} \leq 7 \mathrm{ps}$ and $\leq 6 \mathrm{ps}$ measured for the $1_{1}^{-}$and $3_{1}^{-}$members of the $\mathrm{K}=0^{-}$ band, respectively, result in the $B(E 1)$ rates of $\geq 5 \times 10^{-4} \mathrm{e}^{2} \mathrm{fm}^{2}$, which are surprisingly fast. Clearly, there is no major drop in the B(E1) strength between ${ }^{227} \mathrm{Ra}$ and ${ }^{228} \mathrm{Ra}$. The new results are consistent with some of the predictions for this nucleus and reveal octupole correlation features in ${ }^{228} \mathrm{Ra}$.

We have disproved the previous estimate of $D_{0}=0.011(1)$ e.fm quoted in ref. [12] for ${ }^{228} \mathrm{Ra}$. The lifetimes of the $1_{1}^{-}$and $3_{1}^{-}$states are clearly very short, but it would be within our technical means to improve the precision (or lower the lifetime limit) by a factor of about 3 to 4 . Much tighter limits on the lifetime results would provide a critical verification of the theoretical predictions for the low excitation and low spin states in ${ }^{228} \mathrm{Ra}$. The $\mathrm{B}(\mathrm{E} 1)$ rates may change as a function of excitation energy and spin. However, 
a recent investigation [35] of the high spin states in ${ }^{228} \mathrm{Ra}$ via multinucleon transfer between ${ }^{136} \mathrm{Xe}$ and ${ }^{232} \mathrm{Th}$, yields $\mathrm{D}_{0} / \mathrm{Q}_{0}$ values extracted from the intensity ratios of $\mathrm{E} 1$ and $\mathrm{E} 2$ transitions. For the $\mathrm{I}^{\pi}=11^{-}$state a preliminary value of $\mathrm{D}_{0} / \mathrm{Q}_{0}=0.92(15) \times 10^{-4} \mathrm{fm}^{-1}$ was obtained [35], which implies a $\mathrm{D}_{0}$ of $0.07(1) \mathrm{e} \cdot \mathrm{fm}$. A combination of our results with this value may indicate a constancy of the $\mathrm{B}(\mathrm{E} 1)$ strength from the low spin to the medium spin states in ${ }^{228} \mathrm{Ra}$.

\section{$5 \quad$ Acknowledgements}

This work was supported in part by the Polish Scientific Research Committee under grant KBN-2-P03B-001-11, the Swedish Natural Science Research Council, the Norwegian Research Council, the Spanish C.I.C.Y.T. under contracts AEN94-0833-C02-02 and AEN96-1662, the Canadian Natural Sciences and Engineering Research Council, and UK Engineering and Physical Science Research Council. One of us (K.G.) would also like to thank the OSIRIS group for their generous hospitality and financial support during the stay at Studsvik.

\section{References}

[1] W. Kurcewicz, I.S. Grant, K. Gulda, A.J. Aas, J. Billowes, M.J.G. Borge, D.G. Burke, P.A. Butler, J.F.C. Cocks, B. Fogelberg, S.J. Freeman, G.D. Jones, E. Hagebø, P. Hoff, J. Hønsi, A. Lindroth, G. Løvhøiden, H. Mach, T. Martinez, R.A. Naumann, K. Nybø, G. Nyman, H. Ravn, B. Rubio, J.Simpson, A.G. Smith, J.F. Smith, K. Steffensen, J.L. Tain, O. Tengblad, T.F.Thorsteinsen and the ISOLDE Collaboration, Nucl. Phys. A621 (1997) 827.

[2] A.J. Aas, H. Mach, M.J.G. Borge, B. Fogelberg, I.S. Grant K. Gulda, E. Hagebø, W. Kurcewicz, J. Kvasil, A. Lindroth, T. Martinez, D. Nosek, B. Rubio, J.F. Smith, K. Steffensen, J.L. Tain, O. Tengbald, T.F. Thorsteinsen and ISOLDE Collaboration, Nucl. Phys. A611 (1996) 281.

[3] P.A. Butler and W. Nazarewicz, Rev. Mod. Phys. 68 (1996) 349.

[4] J.L. Egido and L.M. Robledo, Nucl. Phys. A494 (1989) 85.

[5] J.L. Egido and L.M. Robledo, Nucl. Phys. A524 (1991) 65.

[6] P. Möller, J.R. Nix, W.D. Myers and W.J. Swiatecki, At. Data Nucl. Data Tables 59 (1995) 185.

[7] E. Ruchowska, W. Kurcewicz, N. Kaffrell, T. Bjornstad, and G. Nyman, Nucl. Phys. A383 (1982) 1.

[8] A.M. van den Berg, N. Blasi, R.H. Siemssen, W.A. Sterrenburg, and Z. Sujkowski, Nucl. Phys. A422 (1984) 45.

[9] S.A. Ahmad, W. Klemp, R. Neugart, E.W. Otten, P.-G. Reinhard, G. Ulm and K. Wendt, Nucl. Phys. A483 (1988) 244.

[10] R.E. Bell, S. Bjørnholm, J.C. Severiens, Kgl. Danske Videnskab. Selskab, Mat.fys. Medd. 32, No. 12 (1960).

[11] V.Yu. Denisov, Yad. Fiz. 49 (1989) 644.

[12] P.A. Butler and W. Nazarewicz, Nucl. Phys. A533 (1991) 249.

[13] A. Sobiczewski, Z. Patyk, S. Cwiok, and P. Rozmej, Nucl. Phys. A485 (1988) 16.

[14] A. Coc, C. Thibault, H. T. Duong, P. Juncar,S. Liberman, J. Pinard, J. Lerme, J. L. Vialle, S. Buttgenbach, A. C. Mueller, A. Pesnelle, Phys. Lett. 163B (1985) 66.

[15] H. Mach, J. Billowes, M.J.G. Borge, D.G. Burke, P.A. Butler, J.F.C. Cocks, B. Fogelberg, S..J. Freeman, I.S. Grant, K. Gulda, G.D. Jones, E. Hagebø, P. Hoff, J. Hønsi, W. Kurcewicz, G. Løvhøiden, R.A. Naumann, K. Nybø, G. Nyman, H. Ravn, B. 
Rubio, J. Simpson, A.G. Smith, J.F. Smith, K. Steffensen, J.L. Tain, O. Tengblad, T.F. Thorsteinsen and the ISOLDE Collaboration, in Nuclear Shapes and Nuclear Structure at Low Excitation Energy, edited by M. Vergnes, D. Goutte, P.H. Heenen and J. Sauvage (Editions Frontieres, Gif-sur-Yvette, 1994) p. 391.

[16] M.J.G. Borge, D.G. Burke, H. Gietz, P. Hill, N. Kaffrell, W. Kurcewicz, G.G. Løvhøiden, S. Mattsson, R.A. Naumann, K. Nybø, G. Nyman and T.F. Thorsteinsen, Proc. Inter. Conf. Symmetries and Nuclear Structure, Dubrovnik, Yugoslavia, Jun 5-14, 1986, R.A. Meyer, V. Paar. Eds., (Harwood Academic, London, 1986) p. 309.

[17] M.J.G. Borge, D.G. Burke, H. Gietz, P. Hill, N. Kaffrell, W. Kurcewicz, G.G. Løvhøiden, S. Mattsson, R.A. Naumann, K. Nybø, G. Nyman and T.F. Thorsteinsen, Nucl. Phys. A464 (1987) 189.

[18] F. Rösel, H. M. Fries, K. Alder and H. C. Pauli, At. Data and Nucl. Data Tables 21 (1978) 291.

[19] P.C. Sood, D.M. Headly and R.K. Sheline, At. Data and Nucl. Data Tables 51 (1992) 273.

[20] J. Jänecke, F.D. Becchetti, D. Overway, J.D. Cossairt and R.L. Spross, Phys. Rev. C23 (1981) 101.

[21] Y. A. Akovali, Nucl. Data Sheets 71 (1994) 181.

[22] M.J. Martin, Nucl. Data Sheets 49 (1986) 136.

[23] H. Mach, R.L. Gill and M. Moszyński, Nucl. Instrum. Methods A280 (1989) 49.

[24] M. Moszyński and H. Mach, Nucl. Instrum. Methods A277 (1989) 407.

[25] H. Mach, F.K. Wohn, G. Molnár, K. Sistemich, J.C. Hill, M. Moszyński, R.L. Gill, W. Krips and D.S. Brenner, Nucl. Phys. A523 (1991) 197.

[26] H. Mach and B. Fogelberg, Physica Scripta T 56 (1995) 270.

[27] H. Mach et al., to be published.

[28] A.V. Aldushchenkov and N.A. Voinova, Nucl. Data Tables 11 (1972) 299.

[29] A.S. Goldhaber and G. Scharff-Goldhaber, Phys. Rev. C17 (1978) 1171.

[30] H.J. Daley and B.R. Barrett, Nucl. Phys. A449 (1986) 256.

[31] W. Kurcewicz, K. Stryczniewicz, J. Zylicz, S. Chojnacki, T. Morek and I. Yutlandov, Acta Phys. Pol. B2 (1971) 451.

[32] A. Bohr and B.R. Mottelson, "Nuclear Structure" vol. 2 (Benjamin, Massachusetts, 1975) Ch. 6.

[33] M. Gai, Phys. Rev. Lett. 61 (1988) 2424.

[34] H. Mach, B. Fogelberg, M. Sanchez-Vega, A. J. Aas, K. I. Erokhina, K. Gulda, V. I. Isakov, J. Kvasil and G. Lhersonneau, in Physics of Unstable Nuclear Beams, edited by C. A. Bertulani, L. F. Canto and M. S. Hussein (World Scientific, 1997) p. 338.

[35] J. F. C. Cocks et al., to be published. 